\title{
Modification des propriétés du brai de goudron de houille utilisé dans la
}

\section{fabrication des anodes}

Julie Bureau, Duygu Kocaefe*, Yasar Kocaefe, Dipankar Bhattacharyay, Brigitte Morais

Chaire institutionnelle sur les matériaux industriels (CHIMI) et Centre de recherche sur l'Aluminium-REGAL

Université du Québec à Chicoutimi, 555 boulevard de l'Université, Chicoutimi, Québec, G7H 2B1, Canada

Mots clés : Propriétés du brai, Additifs, Mouillabilité, FTIR, Interaction Coke/Brai

\section{RÉSUMÉ}

La fabrication de l’aluminium par le procédé d’électrolyse nécessite l’utilisation des anodes. Or, la qualité des anodes a un impact direct sur le coût du métal, la consommation d'énergie et les émissions environnementales. Une liaison satisfaisante entre le coke et le brai affecte fortement les propriétés finales de l'anode. Une des avenues les plus prometteuses pour améliorer cette liaison est la modification des propriétés du brai. L’objectif de ce travail est d'améliorer la mouillabilité du coke par le brai en modifiant les propriétés du brai par l'ajout de produits chimiques. Des tensioactifs ou agents de modification de surface sont sélectionnés afin d'enrichir les groupements fonctionnels requis pour augmenter la mouillabilité. De plus, les additifs chimiques sont choisis en fonction de l'impact environnemental de même que l'impact économique de cet ajout d'additif pour l'industrie tout en prévenant la contamination des anodes. La méthodologie prévoit d'analyser la composition chimique des brais modifiés par la spectroscopie infrarouge à transformée de Fourier (FTIR) afin d'évaluer l'enrichissement des groupements fonctionnels requis. Puis, l'interaction du brai modifié en présence du coke est vérifiée par la méthode goutte-sessile afin d’en évaluer la mouillabilité du coke par le brai. Une amélioration de la mouillabilité signifie une possible amélioration de l'interaction entre les deux matériaux pouvant améliorer les propriétés anodiques. Dans cette étude, deux des additifs sélectionnés ont permis de démontrer une modification significative des propriétés du brai favorisant l'amélioration de la compatibilité du brai avec le coke.

\section{INTRODUCTION}

Afin d'obtenir l’aluminium primaire en fusion, le procédé électrolytique élaboré par Hall-Héroult emploie de l'alumine $\left(\mathrm{Al}_{2} \mathrm{O}_{3}\right)$ dissoute dans la cryolithe (le bain). ${ }^{[1,2]}$ Le carbone nécessaire à la réduction de l'aluminium est fourni par les anodes. La fabrication d'une anode crue comprend la préparation de la pâte anodique qui se compose des agrégats secs (coke du pétrole calciné, mégot, anode recyclée crue et cuite) (85\%) et d'un liant (brai de goudron de houille) (15\%). ${ }^{[1-3]}$ En pénétrant dans les pores et couvrant la surface du matériel granulaire, le brai procure une cohésion de la pâte anodique. Le matériel est transféré dans un vibrocompacteur afin de former l'anode avant de procéder à la cuisson. Au cours du processus de cuisson des anodes, la décomposition du brai engendre la formation d'une matrice solide par la carbonisation du brai ce qui contribue à l'amélioration de la résistance et de la conductivité électrique des anodes

\footnotetext{
*Duygu_Kocaefe@uqac.ca
} 
produites. ${ }^{[1-4]}$ Par conséquent, la capacité de mouillabilité du brai doit donc être suffisante afin d’interagir adéquatement avec les agrégats secs, plus particulièrement le composant majoritaire, le coke.

La mouillabilité peut se définir comme étant le degré d'étalement d'un liquide sur un solide et dépend de la tension de surface des phases gazeuses, liquides et solides. Lorsqu'une goutte de liquide est déposée à la surface d'un solide, elle adopte une forme caractéristique selon son affinité avec le solide. Généralement, l'étude de la mouillabilité mesure l’angle de contact ce qui correspond au degré de mouillage lorsqu'un solide et un liquide interagissent. ${ }^{[5]}$ La Figure 1 montre que l'angle $\theta$ formé entre les vecteurs associés à l'énergie de l’interface solide/liquide $\left(\gamma_{S L}\right)$ et liquide/vapeur $\left(\gamma_{\mathrm{LV}}\right)$ correspond au mouillage. Plus un angle est petit $\left(<<90^{\circ}\right)$, plus la mouillabilité sera grande. À l'inverse, plus l'angle de contact est élevé $\left(>>90^{\circ}\right)$, plus le mouillage sera considéré comme étant faible. ${ }^{[1,5-7]}$ La relation, à l'état d'équilibre, entre l'angle et les vecteurs d'énergies superficielles $\gamma_{S L}, \gamma_{L V}$ et $\gamma_{S V}$ est donnée par la relation de Young :[1, 6, 7]

$$
\gamma_{S V}=\gamma_{S L}+\gamma_{L V} \cos \theta(1)
$$

Plus spécifiquement, le mouillage dynamique permet d'observer le comportement d'écoulement du brai sur un lit de coke. La mouillabilité dépend alors des propriétés physiques et chimiques du coke (la texture, la porosité, la taille des particules et la composition chimique) et du brai (la composition chimique, le point de ramollissement, la tension superficielle et la viscosité). ${ }^{[5,7]}$ De plus, les groupements fonctionnels à la surface du coke et du brai sont susceptibles d'induire des interactions intermoléculaires entre les molécules du brai et du coke pouvant favoriser la mouillabilité. Plusieurs types d’interactions sont principalement rencontrés entre le coke et le brai. Différents auteurs suggèrent la présence de liaisons hydrogènes, d’interactions électrostatiques, de forces de dispersion, d’interactions acide-base et de liaisons covalentes. ${ }^{\text {[8-11] }}$

De fait, la nature du lien entre le coke et le brai est considérée comme étant le fondement même pour l'obtention des propriétés finales des anodes. ${ }^{[7,12,13]}$ Ainsi, la qualité des anodes a un impact direct sur le coût du métal, la consommation d'énergie et les émissions environnementales. Par conséquent, la compatibilité du coke avec le brai a une importance significative. Toutefois, la qualité du coke et du brai acheminés vers l'industrie sont variables de jour en jour et ne permettent pas toujours d'assurer leurs compatibilités. ${ }^{[14]}$ La qualité décroissante des matières premières mène à l'exploration des différentes solutions. Une des avenues possibles pour 
favoriser la formation du lien est la modification du brai par l'ajout d'un agent de modification de surface ou d'un tensioactif. L'ajout d'additifs chimiques pourrait potentiellement réduire le coût de production, diminuer de la consommation d'énergie et des émissions environnementales ainsi qu'augmenter de la productivité par l'amélioration des propriétés anodiques.

Plusieurs auteurs rapportent différentes méthodes de modifications des propriétés du brai par l'ajout d'additifs. ${ }^{[7,15-19]}$ Oh et Park mentionnent l'usage d'un ajout de soufre élémentaire comme additif chimique pour le traitement du brai. ${ }^{[15]}$ La présence de soufre peut avoir un effet positif ou négatif dans la fabrication des anodes selon sa concentration. ${ }^{[9]}$ Toutefois, son utilisation a un impact environnemental défavorable. ${ }^{[9]}$ L'addition de soufre a permis l'obtention d'une meilleure carbonisation du brai comparativement au brai non modifié. L'ajout induit une augmentation du point de ramollissement, attribué à l'augmentation de la fraction d'insolubles dans la quinoléine (IQ), et une stabilité thermique élevée. ${ }^{[15]}$ Lin et al. ont réalisé la modification de $100 \mathrm{~g}$ de brai de goudron de houille $(\mathrm{BGH})$ avec du divinylbenzène (DVB) en présence d'acide p-toluène sulfonique (PTS) et catalysé par phénol para tert-butyle (PPTB). ${ }^{[17]}$ Les BGHs modifiés obtiennent de 8 à $15 \%$ d'augmentation de la valeur en cokéfaction et une amélioration de la texture optique due à l'amélioration de la texture anisotropique comparativement au brai non modifié. ${ }^{[17]}$ Ren et al. ont réalisé des essais systématiques avec trois différents additifs : le glycéride activée (GA), le sulfate d'aluminium avec des oxydes de polyéthylène (SA-OPE) et le divinylbenzène avec de l'acide p-toluène sulfonique (DVB-APTS) ${ }^{[20]}$ L'ajout de GA ne modifie pas la composition chimique du BGH, mais en augmente tout de même la viscosité induisant une amélioration de la perméabilité de 9,46 à 14,21 \% pour un ajout maximum de $3 \%$ de GA. Le SAOPE agit sur les particules d'IQ primaire permettant la formation d'agrégat de plus grandes particules de gel. La création de particules plus grandes engendre une hausse de la porosité augmentant ainsi le coefficient de perméabilité à un maximum de 16,95 \% de façon non linéaire avec la concentration de SA-OPE. L'ajout de DVB par catalyse de APTS jusqu'à concurrence de 20 \% diminue la perméabilité. Même en augmentant la quantité de DVB, la perméabilité obtenue demeure inférieure au BGH non modifié.

L'objectif de cette étude est d'améliorer la mouillabilité du coke par le brai en modifiant les propriétés du brai par l'ajout d’additifs chimiques. Plus spécifiquement, les additifs choisis doivent être peu coûteux et renfermer des groupements fonctionnels pouvant se lier 
chimiquement au brai. De plus, leurs compositions chimiques doivent pouvoir se lier au coke afin de possiblement favoriser la compatibilité entre le coke et le brai. Le point d'ébullition des additifs doit être inférieure à la température maximale de cuisson afin permettre leurs décompositions lors de la cuisson des anodes tout en contribuant à la valeur en cokéfaction. Les additifs chimiques sélectionnés et leurs produits de décomposition ne doivent pas contenir d'atome nuisible au procédé d'électrolyse et donc prévenir la contamination des anodes et au moins maintenir la pureté de l'aluminium. Dans un premier temps, les brais et les additifs sont caractérisés par spectroscopie infrarouge à transformée de Fourier (FTIR). Les brais sont ensuite modifiés et leur composition chimique est comparée à l'aide de la méthode FTIR. Enfin, l'interaction entre le brai et le coke est étudiée grâce à la mouillabilité par la méthode gouttesessile. L'analyse des résultats permet d'évaluer l'effet de la modification des propriétés du brai sur la mouillabilité du coke par le brai dans le but de fabriquer des anodes.

\section{EXPÉRIMENTAL}

\section{Matériel}

Dans cette étude, quatre brais de goudron de houille (Brai 1, Brai 2, Brai 3 et Brai 4) provenant de l'industrie de l'aluminium ont été employés tels que reçus. Les brais ont été modifiés avec un tensioactif (Additif 1) ou deux agents de modification de surface (Additif 2 et Additif 3) achetés chez Alfa Aesar. Les additifs ont été ajoutés selon quatre différentes concentrations (ci) représentant la masse d'additif sur la masse de l'échantillon (g/g), soit 0,005 g/g < c1 < c2 < c3 < c4 $\leq$ 0,05 g/g. Le Tableau 1 présente quelques propriétés des additifs employés. Enfin, trois cokes issus de l'industrie de l'aluminium ont été employés afin d'étudier leur interaction avec les brais (non modifiés et modifiés), soit un coke standard (Coke 1) et un dont la teneur en soufre est faible (Coke 2) et l'autre élevée (Coke 3). Les cokes ont été broyés, puis tamisés afin de conserver les particules entre 100 et $125 \mu \mathrm{m}$ afin d'obtenir une surface lisse permettant d'approximer l'équation de Young (Équation (1)). ${ }^{[4]}$

\section{Caractérisation des brais et des agents chimiques}

Les structures chimiques d'échantillons de brai non modifié et d'agents chimiques purs ont été analysées par spectroscopie infrarouge à transformée de Fourier (FTIR) ambiante. Une pastille de bromure de potassium ( $\mathrm{KBr}$, grade FTIR) contenant 0,01 g/g d'analyte est placée dans le spectromètre Nicolet 6700 FTIR. L'acquisition du spectre s'effectue dans un nombre d'ondes 
compris entre 399 et $4000 \mathrm{~cm}^{-1}$ à raison de 26 balayages par échantillon. La résolution d'enregistrement est de $4 \mathrm{~cm}^{-1}$. L'expérimentation a été répétée à trois reprises pour chacun des échantillons. Un blanc est enregistré avant d'effectuer une série d'analyse afin de diminuer l'effet de l'environnement sur l'analyse. Le logiciel Omnic32 version 7,3 a été utilisé pour l'analyse des spectres représentant la moyenne des trois essais. Les spectres IR de chaque expérimentation ont été transformés en spectres d'absorbance. Puis l'ensemble des spectres a été corrigé en effectuant une ligne de base tracée à partir du point le plus bas à $399 \mathrm{~cm}^{-1}$ jusqu'au point le plus bas à 4000 $\mathrm{cm}^{-1}$. Les aires sous la courbe ont été obtenues grâce à un programme développé par MATLABversion 2008b. La programmation inclut la correction des valeurs en corrigeant l'absorbance de chacun des tests à une concentration exacte de $0,01 \mathrm{~g} / \mathrm{g}$ d'analyte/KBr. Les résultats constituent la moyenne des intégrations des analyses des trois expérimentations.

\section{Modification du brai}

Les brais sont modifiés en ajoutant préalablement l’additif sélectionné dans une éprouvette, puis le brai, selon les concentrations présentées au Tableau 2 pour une masse totale de 20 g. L’éprouvette est placée dans un socle de métal au centre d'une enceinte isolée, sous atmosphère d'azote $\left(\mathrm{N}_{2}\right)$ afin de prévenir l'oxydation du brai au-delà du point de ramollissement $\left(\approx 120^{\circ} \mathrm{C}\right)$. L'enceinte est chauffée avec un pistolet thermique (Master Appliance corp. Model VT-750C). La température interne du brai est mesurée à l'aide d’un thermocouple relié à un thermomètre électronique (Fluke 52 II). Le système atteint une température de $170{ }^{\circ} \mathrm{C}$ en environ trois minutes, soit la température habituellement utilisée en industrie correspondant à $50^{\circ} \mathrm{C}$ au-dessus du point de ramollissement. Puis la température est maintenue entre 170 et $190{ }^{\circ} \mathrm{C}$ pendant une minute durant l'agitation manuelle du mélange. Le tube est alors refroidi sous flux d'azote pendant environ cinq minutes afin d'abaisser le brai modifié sous le point de ramollissement. Lorsque le mélange est à température ambiante, l'éprouvette est cassée afin de récupérer le brai solide (exempt de verre). L’homogénéité du brai modifié est vérifiée par l'analyse FTIR. Des prélèvements du mélange en plusieurs endroits de l’éprouvette sont analysés pour chacun des additifs chimiques (Additif 1 (A1), Additif 2 (A2) et Additif 3 (A3)) ajoutés au Brai 1 (B1) selon une concentration c4, soit les brais modifiés : A1c4B1, A2c4B1 et A3c4B1 (Tableau 2). La méthode de chauffage pour la modification du brai a aussi été appliquée aux brais non modifiés sans ajouter de tensioactifs ou d'agents de modification de surface de sorte que la modification du brai n’influence pas la comparaison des brais non modifiés et modifiés. L’ensemble des brais 
modifiés a été caractérisé par l’analyse FTIR selon la méthode employée pour les brais non modifiés.

\section{Analyse FTIR des brais modifiés}

Les brais modifiés ont été caractérisés par FTIR selon la méthode employée pour les brais non modifiés. Dans cette étude, l'analyse FTIR permet de déterminer l'évolution de l’intensité des pics associés aux groupes fonctionnels sur les surfaces du brai modifiés par les additifs chimiques. Dans le but de déterminer si un brai est chimiquement modifié, l’absorbance mesurée du brai modifié est comparée à l'absorbance calculée du brai non modifié selon une moyenne pondérée. Par exemple, pour le brai modifié A1c2B3 mesuré, l’absorbance du brai B3 à une longueur d'onde donnée $(\lambda)$ est additionnée à l'absorbance correspondante de l'additif A1 pur, A1c2B3 calculé, selon l'équation :

Absorbance calculée pour A1c2B3 $=\left(\mathrm{Abs}_{\lambda} \times \mathrm{c} 2 / 100\right)_{\mathrm{A} 1}+\left(\mathrm{Abs}_{\lambda} \times(100-\mathrm{c} 2) / 100\right)_{\mathrm{B} 3}(2)$

Ensuite, trois différents ratios ont été calculés, soit l'indice d'aromaticité (Ratio A), l'indice de substitution ortho (Ratio O) et l'indice de la présence d’hétéroatomes (Ratio H). Afin de pouvoir comparer l'ensemble des résultats, les bandes d'absorbances choisies doivent être présentes sur l'ensemble des spectres. Le Ratio A représente l'absorbance des pics aromatiques $\left(\approx 3050 \mathrm{~cm}^{-1}\right)$ par rapport au total des pics aromatiques $\left(\approx 3050 \mathrm{~cm}^{-1}\right)$ et aliphatiques $\left(\approx 2800\right.$ et $\left.2990 \mathrm{~cm}^{-1}\right)$. Le Ratio O correspond au rapport entre l'absorbance des pics de la substitution ortho $\left(\approx 750 \mathrm{~cm}^{-1}\right)$ et l'ensemble des substitutions possibles (ortho, méta et para, soit entre $\approx 700$ et $900 \mathrm{~cm}^{-1}$ ). Pour le Ratio $\mathrm{H}$, est le rapport entre l'absorbance des hétéroatomes et le total des pics aromatiques $(\approx$ $3050 \mathrm{~cm}^{-1}$ ) et aliphatiques $\left(\approx 2800\right.$ et $\left.2990 \mathrm{~cm}^{-1}\right)$. Les bandes entre 3400 et $3600 \mathrm{~cm}^{-1}$, autour de $1700 \mathrm{~cm}^{-1}$ et entre 1000 et $1400 \mathrm{~cm}^{-1}$ contiennent des hétéroatomes. Considérant qu'un même groupement chimique peut générer des pics dans plus d'une de ces régions, seule une de ses bandes doit être sélectionnée dans le calcul du Ratio H. En comparant les résultats pour les différentes bandes, les pics entre 3400 et $3600 \mathrm{~cm}^{-1}$ et autour de $1700 \mathrm{~cm}^{-1}$ ne permettre pas de les mettre en corrélation avec les résultats obtenus pour les tests goutte-sessile. La bande sélectionnée, contenant des hétéroatomes, pour le calcul du Ratio H est donc comprise entre 1000 et $1400 \mathrm{~cm}^{-1}$. Le Ratio H est obtenu en effectuant le rapport de l'absorbance des hétéroatomes sur la majorité des carbones présents dans le brai, soit les aromatiques et les aliphatiques. Ces ratios ont été calculés selon les équations suivantes : 
Ratio A :

$\mathrm{Abs}_{\text {Aromatique }} / \mathrm{Abs}_{\text {Aromatique et aliphatique }}$

Ratio O :

$\mathrm{Abs}_{\text {Substitution ortho }} / \mathrm{Abs}_{\text {Total des substitutions aromatiques }}$

Ratio H :

$\mathrm{Abs}_{\text {Hétéroatome }} / \mathrm{Abs}_{\text {Aromatique et aliphatique }}$

Les ratios sont obtenus pour l'ensemble des brais non modifiés, des brais modifiés, ainsi que des brais non modifiés incluant les additifs chimiques purs correspondant à différentes modifications effectuées (Tableau 2). Les résultats des brais modifiés mesurés expérimentalement, pour chacun des ratios, sont comparés avec les ratios calculés du brai non modifié et de l’additif associé. Une différence significative entre les absorbances mesurées et calculées implique une modification de la structure chimique. Le changement de la composition chimique du brai signifie que la surface du brai est modifiée. Conséquemment, la modification du brai indique la présence possible de nouvelles fonctions chimiques en surface pouvant améliorées l'interaction entre le brai modifié avec le coke.

\section{Test goutte-sessile}

La compatibilité entre le brai et le coke a été étudiée à l’aide de la méthode goutte-sessile. Tel que mentionné, les agrégats secs qui entrent dans la composition de l’anode sont constitués de coke de pétrole frais, de mégot et de rejets d'anode crus et cuits. Toutefois, les impuretés contenues dans le mégot ne peuvent être maintenues constantes affectant par le fait même la composition des rejets d’anode. Par conséquent, seul le coke est employé afin de diminuer l’influence des impuretés sur les résultats des tests. Les expérimentations goutte-sessile sont réalisées avec le système expérimental développé à l'UQAC tel que décrit par Sarkar et al. en 2014. ${ }^{[4]}$ La méthode consiste à compacter la poudre de coke dans le creuset d'échantillon pour former un lit de coke dont la surface est lisse. L’échantillon de brai solide (non modifié ou modifié) est placé dans la chambre d'injection. Cette chambre a un petit trou au fond et est placée juste au-dessus de l'échantillon de coke au cours de l'expérience. Les expériences ont été conduites sous atmosphère d'azote $\left(\mathrm{N}_{2}\right)$. Une ligne de $\mathrm{N}_{2}$ est reliée à la chambre d'injection. La pression de cette ligne est supérieure à celle du système afin de pressuriser légèrement la chambre. L'ouverture de la valse reliée à cette ligne force la sortie d’une goutte de brai liquide vers l'échantillon solide. Les expériences de goutte-sessile ont été effectuées à $170{ }^{\circ} \mathrm{C}$. La goutte de brai fondu est déposée directement sur le substrat de coke en tournant la chambre d’injection 
selon la position du trou. Une vidéo de la goutte est captée jusqu'à l'obtention d'un mouillage total. Le système peut capturer des images statiques et dynamiques. Pour mesurer l'angle de contact, le logiciel FTA 32 est utilisé. Pour chaque expérience, l'angle de contact a été pris comme la moyenne des angles mesurés sur deux côtés de la goutte. Chaque expérience a été répétée deux fois et le résultat final représente la moyenne de ces deux expérimentations. Quatre brais non modifiés différents et trois cokes différents ont été expérimentés à l'aide du système goutte-sessile. De même, plusieurs brais modifiés ont été étudiés avec, en totalité ou en partie, les trois cokes sélectionnés. La mouillabilité des échantillons dépend de l'interaction entre le brai en contact avec la surface des particules de coke. Une amélioration de la mouillabilité grâce à un additif chimique pour l'ensemble des brais ayant une composition chimique différente avec plusieurs cokes de nature différente signifie une amélioration possible pour l'ensemble des brais reçu par l'industrie.

\section{RÉSULTATS ET DISCUSSION}

\section{Analyse FTIR des brais}

Les groupements fonctionnels à la surface des échantillons ont été identifiés par FTIR. La Figure 2, la Figure 3 et la Figure 4 présentent les spectres mesurés du brai B1 non modifié et des brais B1 modifiés avec, respectivement, les additifs A1, A2 et A3 à différentes concentrations (c1, c2 et c4), ainsi que le spectre calculé du brai B1 additionné de chacun des additifs pour la concentration c4. Plusieurs groupements fonctionnels sont observables sur les spectres FTIR des brais. La bande située à 3000 et $3100 \mathrm{~cm}^{-1}$ correspond à une élongation de $\mathrm{C}-\mathrm{H}$ aromatique. ${ }^{[21]}$ Alors que les pics situés entre 2800 et $2980 \mathrm{~cm}^{-1}$ correspondent à une liaison C-H aliphatique. ${ }^{[21]}$ Les pics situés dans la bande comprise entre 1400 et $1660 \mathrm{~cm}^{-1}$ sont associés aux alcanes (C-C) ou alcènes $(C=C)$. Plus spécifiquement, les $C-C$ se retrouvent entre 1400 et $1475 \mathrm{~cm}^{-1}$ sous forme de pics forts, les $\mathrm{C}=\mathrm{C}$ présentent des pics forts à 1400 et $1455 \mathrm{~cm}^{-1}$ et moyens, s'ils sont conjugués, à 1620 et $1660 \mathrm{~cm}^{-1}$, tandis que les $\mathrm{C}=\mathrm{C}$ aromatiques auront des pics moyens-forts compris entre 1450 et $1650 \mathrm{~cm}^{-1} \cdot{ }^{[21]}$ Les pics présents dans la bande à 1000 et 1400 se rapportent aux hétéroatomes : ester, éthers, alcool, phénols, amines, etc. ${ }^{[21]}$ Enfin, la bande entre 700 et 900 $\mathrm{cm}^{-1}$ est associée aux substitutions de l'anneau aromatique. ${ }^{[21]}$ Seules les expérimentations du brai B1, modifié avec l'additif A1 pour une concentration c2 et plus, présentent un pic compris entre 1660 et $1820 \mathrm{~cm}^{-1}$. Ce pic est associé au groupement fonctionnel d'un carbonyle ( $\left.\mathrm{C}=\mathrm{O}\right)$ provenant de l'ajout de l'additif A1 qui possède un groupement ester. ${ }^{[21]}$ Dû à la présence de 
groupements voisins, le pic du $\mathrm{C}=\mathrm{O}$ peut varier de position. Pour cette raison, un nombre d'onde spécifique pour ce pic n'a pas été utilisé pour l'analyse. L’ensemble des pics présents entre 1660 et $1820 \mathrm{~cm}^{-1}$ a donc été considéré.

\section{Brai B1 modifié avec l'additif A1}

Les spectres d'absorbance, présentés à la Figure 2, et les Ratios $\mathrm{A}$, $\mathrm{O}$ et $\mathrm{H}$ présentés au Tableau 3 du brai B1 et des brais modifiés avec l'additif A1, un tensioactif, montrent une différence significative des résultats mesurés expérimentalement. L'augmentation de la concentration de l'additif A1 entraîne une diminution progressive importante pour le Ratio A et une diminution moins marquée pour le Ratio $\mathrm{H}$, ainsi qu'une augmentation du Ratio O. Toutefois, l'additif A1 contient plusieurs hétéroatomes dans sa composition chimique. À l'inverse des résultats mesurés expérimentalement, l'augmentation de la concentration augmente le Ratio $\mathrm{H}$ pour les résultats calculés. De plus, en comparant les Ratios A mesurés et calculés, la diminution du ratio décroit plus rapidement avec l'accroissement de la concentration pour les brais modifiés (mesurés). Ces résultats expérimentaux permettent d'affirmer que la composition chimique du brai B1 a été modifiée par l'additif A1. La modification du brai B1 avec l'additif A1 augmente le nombre de chaînes aliphatiques libres diminuant ainsi le Ratio A. La tête polaire de l'additif A1, composée d'un ester d'acide carboxylique, s'est possiblement liée au brai B1 en réagissant avec un carbone ayant un dipôle positif dû à la présence d'un hétéroatome ou d'une délocalisation d'électrons $\Pi$. Par conséquent, la queue du tensioactif A1 est donc disponible pour interagir avec le coke. Habituellement, les chaînes aliphatiques libres inhibent le mouillage dû à l'encombrement stérique. Cependant, ces chaînes pourraient favoriser les réactions de condensation. De plus, en présence de chaleur, les chaînes aliphatiques peuvent conduire à des réactions de cyclisation intramoléculaire. Ces réactions sont habituellement importantes lors de la calcination puisqu'elles augmentent le rapport carbone-hydrogène. D’ailleurs, ces réactions sont favorisées par l'accroissement de la substitution ortho (Ratio O). Donc, il est possible que l'augmentation de la concentration de l'additif A1 augmente l'interactivité du brai avec le coke. Une augmentation de l’interactivité pourrait alors augmenter la mouillabilité entre ces deux matériaux.

\section{Brai B1 modifié avec l'additif A2}

Les spectres d'absorbance du brai B1 modifié avec l'additif A2 sont donnés à la Figure 3. Les Ratios $\mathrm{A}, \mathrm{O}$ et $\mathrm{H}$ (calculés et mesurés) obtenus à partir de ses spectres sont présentés au Tableau 
4. En examinant les résultats, il est possible de constater que les ratios calculés et mesurés sont semblables. En effet, l'augmentation de la concentration de l'additif A2 entraine une légère augmentation du Ratio A, mais peu significative ainsi qu'aucune variation significative des Ratios $\mathrm{O}$ et H. L'analyse FTIR ne permet pas d'affirmer que la surface du brai B1 a été modifiée chimiquement par l'additif A1. Par conséquent, il est possible que les groupements alcools ne soient pas accessibles pour se lier au brai. En effet, il est probable que les atomes d'oxygène de la fonction alcool ont interagi avec les atomes d'hydrogène appartenant à d'autres fonctions d'alcool de l'agent de modification de surface pour former une liaison hydrogène. De plus, le brai se compose généralement de molécules de grandes tailles et les fonctions alcools de l'additif A2 sont situées sur de longues chaînes aliphatiques. De sorte que la taille des chaînes aliphatiques de l'additif A2 en présence des molécules de brai provoque probablement un encombrement stérique défavorable à l'interaction avec les groupements fonctionnels situés à la surface du brai. L'ajout de l'additif A2 ne pouvant se lier au brai, le brai n’est alors pas modifié chimiquement. De plus, la présence des longues chaines aliphatiques du mélange peut nuire à l'interaction du brai et du coke. Ainsi donc, l'ajout de l'additif A2 n’améliore probablement pas l'interactivité du brai B1 avec un coke.

\section{Brai B1 modifié avec l'additif A3}

Les spectres d'absorbance, présentés à la Figure 4, du brai B1 et des brais modifiés avec l'additif A3 présentent des courbes similaires sauf vers $1640 \mathrm{~cm}^{-1}$ correspondant à des liaisons $\mathrm{C}=\mathrm{C}$ conjuguées. L’intensité du pic mesuré croit avec l'augmentation de la concentration de l'additif dans le brai modifié. Au Tableau 5, les Ratios A, O et H mesurés du brai B1 et des brais modifiés avec l'additif A3 montrent que les ratios tendent à augmenter de façon peu significative avec l'augmentation de la concentration. De plus, les Ratios A et O mesurés et calculés présentes des résultats similaires. Toutefois, le Ratio $\mathrm{H}$, des brais calculés, diminue avec l'accroissement de la concentration de l'additif A3. Selon le calcul établi à l’Équation 2, l'absorbance calculée du brai est fonction de la masse de brai par rapport au total de l'échantillon. Conséquemment, le nombre

d'hétéroatomes par rapport aux hydrogènes aromatiques et aliphatiques (Équation 5) contenus dans l'additif A3 est possiblement inférieur au ratio présent dans le brai B1, de sorte que le Ratio H calculé diminue avec l'augmentation de la concentration de l'additif A3. L'écart entre les résultats mesurés et calculés pour le Ratio $\mathrm{H}$ démontre que la composition chimique du brai B1 a été modifiée par l’additif A3 en augmentant le ratio d'hétéroatomes présents. De plus, les 
différents groupements fonctionnels de l'additif A3 ont tous la possibilité de réagir avec les groupements fonctionnels à la surface du brai. Des analyses supplémentaires, par exemple la spectrométrie photoélectronique induite par rayon $\mathrm{X}$, seraient nécessaires afin d'établir comment l'additif A3 se lie au brai. Néanmoins, l'augmentation de l'ensemble des ratios tend à démontrer une possible augmentation de l'ensemble des types d'interactions possibles entre le brai et le coke. Donc, la modification du brai B1 avec l'additif A3 pourrait améliorer la mouillabilité du coke par le brai modifié.

Étant donné que la mouillabilité et les résultats FTIR avec tous les brais et les additifs ont montré des tendances similaires, seuls les résultats FTIR des expérimentations menées avec le brai B1 sont présentés dans cet article.

\section{Analyses des tests de mouillabilité}

L'analyse par test goutte-sessile permet de comparer le comportement de mouillage du coke par le brai en observant l'angle de contact en fonction du temps. Afin de visualiser l'effet de l'ajout d'additifs chimiques au brai comparativement à un brai non modifié, la forme des gouttes de brai fondu sur un lit de coke, pour une même concentration d'additif, peut être révélateur. La Figure 5 montre certaines des images capturées lors de l'enregistrement vidéo durant les tests gouttesessile du brai B1 non modifié et modifié avec une concentration c2 pour chacun des trois additifs sur un lit de particules de Coke 1 à 0 et environ 100 s. Au temps initial, il est possible d'observer que l'angle de contact des gouttes est similaire pour chacune des expérimentations. De plus, un résultat similaire des angles de contact est aussi obtenu à environ $100 \mathrm{~s}$ pour le brai A3c2B1, (Figure 5h) et le brai B1 (Figure 5b). Ce qui permet d'affirmer que cette concentration n’est possiblement pas suffisante pour améliorer l'affinité du brai B1 avec le Coke 1. Par contre, la comparaison de la Figure 5b et de la Figure 5d obtenues à partir des expérimentations avec le brai B1 et le brai A1c2B1 à environ 100 s démontre une diminution de l'angle de contact avec l'ajout de l'additif A1. Une amélioration de l'angle de contact signifie une possible amélioration de l'interaction coke/brai grâce à la modification avec l'additif A1. À l'opposé, la Figure 5b et la Figure 5h captées lors de l'enregistrement du test goutte-sessile à environ $100 \mathrm{~s}$ pour le brai B1 et le brai A2c2B1 démontre que l'angle de contact à augmenter avec l'ajout de l'additif A2. Ce résultat indique que l'additif A2 n'est sans doute pas favorable à l'amélioration de la mouillabilité. 
La Figure 6 présente les angles de contact dynamique du brai B1 non modifié et quelques-unes des modifications effectuées en fonction du temps avec le Coke 1. Il est possible d'observer que les courbes décroissent de façon proportionnelle jusqu'à pénétration totale. Pour chacune des expérimentations réalisées, l'angle de contact initial est similaire. À noter que les tests de mouillabilité ne sont pas réalisés sur une surface complètement solide. Néanmoins, de petites particules de coke sont compactées pour former un lit de coke afin d'approximer une surface lisse lors de chaque expérimentation. Étant donné la nature de la surface, les résultats des tests gouttesessile sont affectés par deux phénomènes, soit: le mouillage et la pénétration. De plus, ces deux phénomènes sont également liés, car si le liquide ne mouille pas le solide, il ne pénètre pas dans le lit de coke. Par conséquent, les différentes expérimentations seront comparées selon le temps de pénétration, car un meilleur mouillage entraînera des temps de pénétration plus courts.

Dans cette étude, l'ensemble des brais non modifiés ont été analysé. Les brais B2, B3 et B4 ont tous des temps de pénétration similaire tels que présentés au Tableau 6. Ces brais ont donc été classés, en ordre croissant, selon leur capacité à diminuer le temps de pénétration lorsqu'ils sont modifiés. L'observation des résultats obtenus permet d'affirmer que la modification des propriétés des brais a diminué le temps de mouillage total grâce à l'additif A1 ou l'additif A3. Une concentration de c1 de l'additif A1 est suffisante afin d'améliorer le temps de mouillage pour le brai B1, tandis qu'une concentration minimale de c3 est requise pour l'additif A3. À l'opposé, la modification du brai B1 avec l'additif A2 a augmenté le temps de mouillage total. Puisque l'additif A2 n'a pas permis l'amélioration des propriétés ayant la moins bonne mouillabilité (brai B1), l'effet de cet ajout d'additif n’a pas été étudié davantage. Enfin, le brai B1 non modifié possède un temps de pénétration supérieur aux trois autres brais. Donc, le brai B1 permet possiblement moins de liaisons avec le Coke 1 et est probablement moins compatible. Par conséquent, les analyses goutte-sessile ont principalement porté sur la modification du brai B1 avec les deux autres additifs.

De façon plus générale, il est possible d'affirmer que la modification des brais par l'ajout des additifs A1 ou A3 a pour effet de diminuer le temps de mouillage comparativement au brai non modifié (Figure 6 et Tableau 6). De plus, l'augmentation de la concentration d'additif diminue le temps de mouillage total. Conséquemment, l'interaction du brai B1 modifié avec l'additif A1 ou A3 augmente avec l'augmentation de la concentration d'additif. En comparant les temps de 
mouillage total des additifs A1 et A3 de même concentration (Tableau 6), il est possible d'observer que l'effet est plus important pour l'additif A1. Le brai modifié avec l'additif A1 permet possiblement plus d'interaction avec le Coke 1 comparativement avec le brai modifié par l'additif A3. Cependant, des similarités sont observables entre ces deux additifs. En effet, les temps de pénétration de l'additif A3 pour une concentration de c3 et c4 sont respectivement similaires à ceux de l'additif A1 pour une concentration de c1 et c2.

La Figure 7 et la Figure 8 présentent les résultats des tests goutte-sessile du brai B1 pour deux différents cokes, le Coke 2 à basse teneur en soufre (Figure 7) et le Coke 3 (Figure 8) à haute teneur en soufre. Bien que ces deux cokes soient de mêmes granulométries que le Coke 1, la forme des particules n'est pas nécessairement la même. Puisque la forme des particules a une influence sur la compaction sur la formation du lit de coke, le temps de pénétration sera lui aussi influencé. De sorte que les résultats de la mouillabilité obtenus pour un brai modifié doivent être comparés uniquement avec le brai non modifié pour un même coke.

En observant les courbes des deux graphiques, il est possible de constater que plus la concentration en additif augmente, plus le temps de pénétration total diminue. De plus, la modification du brai par l'additif A1 a une capacité de mouillage accrue pour une même concentration de l'additif A3. Ces résultats sont conformes à ceux obtenus pour le Coke 1. Conséquemment, la modification du brai B1 par l'additif A1 ou A3 permet d'améliorer la mouillabilité pour l'ensemble des cokes analysés. D’ailleurs, il a été démontré que la modification de quatre brais différents avec l'additif A1 ou A3 peut diminuer le temps de pénétration total. Dès lors, il est probable que les additifs A1 et A3 modifient la surface des brais de sorte qu'ils améliorent l'interaction avec des cokes, et ce peu importe les matériaux de base.

Ce projet est réalisé dans le cadre d'une maîtrise en ingénierie à l'Université du Québec à Chicoutimi et constitue la première étape de l'étude. La suite des travaux prévoit la fabrication d'anodes de laboratoire afin de vérifier l'effet de la modification des brais par l'ajout d'additifs.

\section{CONCLUSIONS}

Dans ce projet, l'effet de la modification de brais par l'ajout de trois différents additifs a été étudié à l'aide de la méthode FTIR et du test goutte-sessile. Tout d'abord, la composition chimique des brais non modifiés et modifiés a été comparée par la méthode FTIR. Ensuite, 
l'interaction entre le brai modifié et le coke a été évaluée par mouillabilité. Les conclusions suivantes peuvent être formulées à partir de l'analyse des résultats obtenus.

Tout d'abord, la composition chimique des brais non modifiés et modifiés a été comparée par la méthode FTIR. L'analyse des résultats obtenus pour la modification par l'ajout de l'additif A1 indique une diminution de l'indice d'aromaticité et une augmentation du nombre de substitutions en position ortho. L'additif A1 est un tensioactif dont la tête polaire se lie probablement au brai et dont la queue hydrophobe est disponible pour se lier au coke. Dès lors, la diminution du ratio d'aromatique est probablement attribuable à l'augmentation du nombre de chaînes aliphatiques présentes par rapport au nombre des composés aromatiques. La présence de ses chaînes aliphatiques peut favoriser les réactions de condensation et de cyclisation intramoléculaire favorable à une augmentation de la valeur en cokéfaction. L'analyse des résultats pour la modification par l'ajout de l'additif A2 ne permet pas de démontrer que le brai a été modifié chimiquement. Enfin, l'analyse des résultats de la modification du brai par l'additif A3 indique que le brai non modifié a été transformé chimiquement en augmentant la présence d'hétéroatomes. Une augmentation de la présence d'hétéroatomes signifie une augmentation possible du nombre de liaisons hydrogènes, d'interactions électrostatiques, d'interactions acidebase et de liaisons covalentes entre le brai et le coke. Par conséquent, la méthode FTIR a permis de démontrer que l'ajout de l'additif A1 ou A3 peut modifier chimiquement la composition du brai, tandis qu'aucun changement significatif n’est observé avec l'additif A2. L'influence de la modification chimique des brais a été évaluée par la mouillabilité du coke par le brai. Les résultats des tests goutte-sessile démontrent une amélioration du mouillage entre le coke et le brai modifié avec l'augmentation de l'ajout des additifs A1 et A3. À l'inverse, l'ajout d'additif A2 démontre une diminution du mouillage entre le coke et le brai modifié. Par conséquent, il est possible que seuls les additifs A1 et A3 améliorent les interactions entre le brai et le coke.

Cette étude a permis de démontrer qu'il est possible de modifier chimiquement les propriétés du brai afin d'améliorer sa mouillabilité avec le coke en utilisant des tensioactifs ou agents de modification de surface. Les additifs chimiques A1 et A3 ont permis de réduire le temps de mouillage ce qui pourrait contribuer à améliorer l'adhésion du brai au coke lors de la fabrication des anodes. Étant donné la composition chimique des additifs employés, celle-ci ne contaminera pas les anodes fabriquées. De plus, leurs produits de décomposition pourraient contribuer à 
augmenter la valeur en cokéfaction lors de la cuisson des anodes et ne nuiront pas au processus de fabrication de l'aluminium.

\section{REMERCIEMENTS}

Nous tenons à remercier vivement le soutien du Conseil de recherches en sciences naturelles et en génie du Canada (CRSNG) et de l'Université du Québec à Chicoutimi (UQAC).

\section{NOMENCLATURE}
Abs
Absorbance
PTS
Acide p-toluène sulfonique
$\theta$
Angle
$\mathrm{Ai}$
Additif (où i = 1, 2 ou 3)
$\mathrm{Bi}$
Brai (où i = 1, 2, 3 ou 4)
BGH Brai de goudron de houille
C Calcul obtenu par la moyenne pondérée
ci Concentration (où i $=1,2,3$ ou 4) (g/g)
DVB Divinylbenzène
DVB-APTS Divinylbenzène avec de l'acide p-toluène sulfonique
GA Glycéride activée
Ratio A Indice d'aromaticité
Ratio O Indice de substitution ortho
Ratio H Indice de la présence d’hétéroatomes
M Mesuré expérimentalement
PPTB Phénol para tert-butyle (PPTB)
QI Quinoléine
FTIR Spectroscopie infrarouge à transformée de Fourier
SA-OPE Sulfate d'aluminium avec des oxydes de polyéthylène
$\gamma_{\mathrm{LV}} \quad$ Tension superficielle des interfaces liquide/vapeur $(\mathrm{mN} / \mathrm{m})$
$\gamma_{\mathrm{SL}} \quad$ Tension superficielle des interfaces solide/liquide $(\mathrm{mN} / \mathrm{m})$
$\gamma_{\mathrm{SV}} \quad$ Tension superficielle des interfaces solide/vapeur $(\mathrm{mN} / \mathrm{m})$ 


\section{RÉFÉRENCES}

1. A. Charette, Y. S. Kocaefe, D. Kocaefe, Le carbone dans l'industrie de l'aluminium. xxi ed, Les presses de l'aluminium, Chicoutimi, Québec 2012, p. 325.

2. K. L. Hulse, Anode manufacture : raw materials, formulation and processing parameters. xxxv ed, R \& D Carbon Ltd., Sierre, Suisse 2000, p. 416.

3. A. D. Kamran, Investigation of the materials and paste relationships to improve forming process and anode quality, Ph.D. thesis, Université Laval, Québec, Canada 2013, p. 148.

4. A. Sarkar, D. Kocaefe, Y. S. Kocaefe, D. Sarkar, D. Bhattacharyay, B. Morais, J. Chabot, Fuel. Coke-pitch interactions during anode preparation. 2014, 117 (PART A), 598.

5. A. Sarkar, D. Kocaefe, Y. Kocaefe, D. Bhattacharyay, D. Sarkar, B. Morais, Energy \& Fuels. Effect of Crystallinity on the Wettability of Petroleum Coke by Coal Tar Pitch. 2016, 30 (4), 3549.

6. J. Schultz, Z. Kessaissia, Carbon. Étude des phénomènes de mouillabilité du carbone par le brai liquide. 1982, 20 (5), 395.

7. V. G. Rocha, C. Blanco, R. Santamaría, E. I. Diestre, R. Menéndez, M. Granda, Fuel. Pitch/coke wetting behaviour. 2005, 84 (12), 1550.

8. D. Kocaefe, A. Sarkar, S. Das, S. Amrani, D. Bhattacharyay, D. Sarkar, Y. S. Kocaefe, B. Morais, M. Gagnon, TMS Light Metals. Review of different techniques to study the interactions between coke and pitch in anode manufacturing. 2013, 1045.

9. U. Suriyapraphadilok, Characterization of Coal- and Petroleum-derived Binder Pitches and the Interaction of Pitch/coke Mixtures in Pre-baked Carbon Anodes, Thèse de doctorat, Pennsylvania State University, State College 2008, p. 354.

10. X. Huang, D. Kocaefe, Y. Kocaefe, D. Bhattacharyay, Colloids and Surfaces A: Physicochemical and Engineering Aspects. Wettability of bio-coke by coal tar pitch for its use in carbon anodes. 2016, 490, 133.

11. A. Sarkar, Effet des propriétés du coke sur les propriétés d'anodes, Thèse de doctorat, Université du Québec à Chicoutimi, Chicoutimi, Québec, Canada 2015, p. 344.

12. J. Cao, A. N. Buckley, A. Tomsett, The Journal of The Minerals, Metals \& Materials Society. Re-examining the pitch/coke wetting and penetration test. 2002, 54 (2), 30.

13. P. Couderc, P. Hyvernat, J. L. Lemarchand, Fuel. Correlations between ability of pitch to penetrate coke and the physical characteristics of prebaked anodes for the aluminium industry. 1986, 65 (2), 281.

14. Réseau Trans-Al, Carte routière technologique canadienne de l'aluminium. 2000, Réseau Trans-Al: Chicoutimi. p. xiv.

15. S. M. Oh, Y. D. Park, Fuel. Comparative studies of the modification of coal-tar pitch. 1999, 78 (15), 1859.

16. V. G. Rocha, M. Granda, R. Santamaría, C. Blanco, E. I. Diestre, R. Menéndez, «Improvement of pitch/coke interactions by addition of surface tension modifiers», Carbon2004, Brown University, Providence, RI, 11-16 July 2004.

17. Q. Lin, T. Li, C. Zheng, Y. Zhao, S. Song, Journal of Analytical and Applied Pyrolysis. Carbonization behavior of coal-tar pitch modified with divinylbenzene and optical texture of resultant semi-cokes. 2004, 71 (2), 817.

18. Q. Lin, T. Li, Y. Ji, W. Wang, X. Wang, Fuel. Study of the modification of coal-tar pitch with p-methyl benzaldehyde. 2005, 84 (2-3), 177. 
19. V. G. Rocha, C. Blanco, R. Santamaría, E. I. Menéndez, R. Granda, M. Diestre, Fuel Processing Technology. The effect of the substrate on pitch wetting behaviour. 2010, 91 (11), 1373.

20. C. Ren, T. Li, F. Song, X. Sun, Q. Lin, Materials Letters. Influence of additives on the permeability of impregnating coal-tar pitch. 2006, 60 (13-14), 1570.

21. N. B. Colthup, J. Opt. Soc. Am. Spectra-Structure Correlations in the Infra-Red Region. 1950, 40 (6), 397. 